\title{
Modelling the Influence of Stresses on Magnetic Characteristics of the Elements of the Truss Using Extended Jiles-Atherton Model
}

\author{
D. JACKIEWICZ ${ }^{a, *}$, R. SzeWCZYK ${ }^{a}$, M. KACHNIARZ ${ }^{b}$ AND R. BIEDRZYCKI ${ }^{c}$ \\ ${ }^{a}$ Institute of Metrology and Biomedical Engineering, Warsaw University of Technology, \\ sw. A. Boboli 8, 02-525 Warszawa, Poland \\ ${ }^{b}$ Industrial Research Institute for Automation and Measurements, Al. Jerozolimskie 202, 02-486 Warsaw, Poland \\ ${ }^{c}$ Faculty of Electronics and Information Technology, Warsaw University of Technology, \\ Nowowiejska 15/19, 00-665 Warsaw, Poland
}

\begin{abstract}
Steel trusses structures are commonly used in civil engineering. Their mechanical parameters, especially the durability, are critical, and often human life depends on it. Therefore it is very important to monitor the critical elements of the truss. For this purpose magnetoelastic method of measurement may be used. It is a method having several advantages over other methods, particularly because of use of elements which are already parts of the structure. The study of structural elements have already been conducted. However, to take advantage of the magnetoelastic characteristics measurement to assess the state of stress, one should have a model of the influence of stress on magnetic properties. The influence of stresses on hysteresis loops was modelled with the extended Jiles-Atherton model. The obtained results of the modelling are consistent with the experimental measurements results. The results of modelling create new possibilities of explanation of the physical phenomena connected with magnetization of the magnetic materials under stresses, which is especially important for the assessment of the state of the constructional steel during its exploitation in industrial conditions.
\end{abstract}

DOI: 10.12693/APhysPolA.131.1189

PACS/topics: 75.60.Ej, 75.85.+t, 81.70.-q

\section{Introduction}

Steel trusses are often used in the construction industry. It is important to monitor their condition and possibly early detect dangerous stresses. Often trusses, especially steel ones, are made of soft magnetic materials. For this purpose magnetoelastic method of measurement may be used. Magnetoelastic effect [1-3] is the change of magnetic properties of the material under the influence of mechanical stresses.

However, to take advantage of the magnetoelastic characteristics measurement to assess the state of stress, quantitative model of stress dependence of the magnetic hysteresis loops is required. A suitable model for modeling the magnetic characteristics under stress in a steel truss is the Jiles-Atherton model.

\section{Experimental}

Measurements of magnetoelastic characteristics were made on test stand shown in Fig. 1. The first part of the test stand is used for measurement of magnetoelastic characteristics. It consists of hysteresisgraph for magnetizing waveforms generation and magnetic flux measurement. Hysteresisgraph is controlled by a PC computer.

\footnotetext{
* corresponding author; e-mail: d.jackiewicz@mchtr.pw.edu.pl
}

The second part of the stand is used for applying stresses. It includes sample truss, force sensor and oil hydraulic press. The truss is made of the $13 \mathrm{CrMo} 4-5$ constructional steel, widely used in the energetic industry.

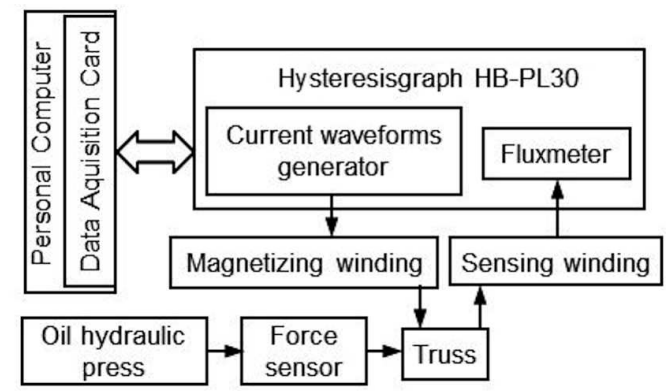

Fig. 1. Schematic block diagram of the measurement stand.

The three middle truss members are the tested samples (Fig. 2). These samples were wound with the magnetizing and sensing windings. With this solution, the magnetic circuit is closed and there is no need to add another measuring elements. The sample was wound by 900 turns of magnetizing winding and 600 turns of sensing winding. The truss was loaded mechanically by oil hydraulic press. The force value was controlled by the force sensor. Measurement of magnetoelastic characteristics were made for amplitudes of magnetizing field $H_{m}$ equal to $350,435,655,870,1310$, and $2180 \mathrm{~A} / \mathrm{m}$. Each 


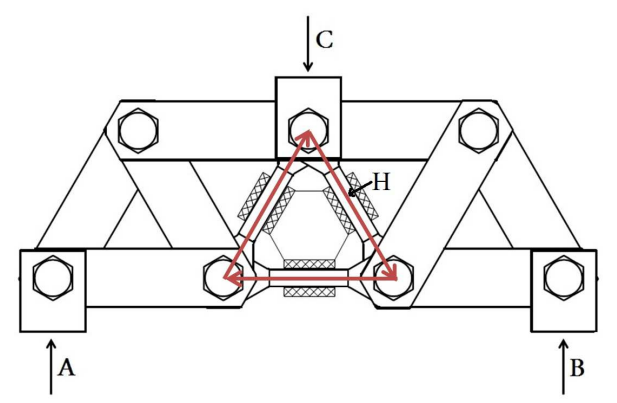

Fig. 2. Schematic of the experimental steel truss structure with marked magnetic circuit. A,B - support points; $\mathrm{C}$ - loading point, $\mathrm{H}$ - magnetic field.

family of the hysteresis loops determined for 17 different values of the applied force $F$ (from $0 \mathrm{kN}$ to $12.7 \mathrm{kN}$ ).

\section{Modeling}

The Jiles-Atherton model of magnetization process [4] is one of the most effective model of magnetic hysteresis loops. This model is based on the concept of anhysteretic magnetization, generalized for isotropic and anisotropic [5,6] materials. In the Jiles-Atherton model, anhysteretic magnetization $M_{a h}$ is given by the set of following equations $[6]$ :

$$
\begin{aligned}
& M_{\text {ah_aniso }}=M_{s}\left(\frac{\int_{0}^{\pi} \mathrm{e}^{(E(1)+E(2)) / 2} \sin \theta \cos \theta \mathrm{d} \theta}{\int_{0}^{\pi} \mathrm{e}^{(E(1)+E(2)) / 2} \sin \theta \mathrm{d} \theta}\right) \\
& E(1)=\frac{H_{e}}{a} \cos \theta-\frac{K_{a n}}{M_{s} \mu_{0} a} \sin ^{2}(\psi-\theta), \\
& E(2)=\frac{H_{e}}{a} \cos \theta-\frac{K_{a n}}{M_{s} \mu_{0} a} \sin ^{2}(\psi+\theta),
\end{aligned}
$$

where case $K_{a n}$ is the average energy density connected with uniaxial anisotropy in a magnetic material, $\psi$ is the angle between direction of the magnetizing field and the easy axis of magnetization due to the anisotropy, $a$ determines domain walls density and $M_{s}$ is saturation magnetization of the magnetic material. It should be indicated that for isotropic magnetic material, where $K_{a n}=0$, Eq. (1) reduces to the Langevin equation [4]. The effective magnetic field $H_{e}=H+\alpha M$ is determined by the total magnetization of material $M$ and interdomain coupling $\alpha$ [4].

Magnetic hysteresis loop in the Jiles-Atherton model is given by the differential equation [4]:

$$
\frac{\mathrm{d} M}{\mathrm{~d} H}=\frac{\delta_{M}}{1+c} \frac{M_{a h}-M}{\delta k-\alpha\left(M_{a h}-M\right)}+\frac{c}{1+c} \frac{\mathrm{d} M_{a h}}{\mathrm{~d} H},
$$

where the parameter $k$ quantifies the average energy required to break pinning site, and $c$ describes reversibility of magnetization process. In this equation, parameter $\delta$ causes hysteretic magnetization and parameter $\delta M$ guarantees that incremental susceptibility is always positive, which is physically justified [7].
One of the most important problem connected with the Jiles-Atherton model is determination of its parameters on the base of experimental hysteresis loops. For this reason presented analyses were divided into two steps. In the first step, the parameters of unstressed sample were determined during the optimization process based on the differential evolution [8] first, and then using the Nelder and Mead simplex optimization. Target function was the sum of squared differences between experimental hysteresis loops and the results of modelling. To achieve better agreement with experiment, the three hysteresis loops measured for the different values of amplitude of magnetizing field were considered simultaneously [8]. Due to the fact that $13 \mathrm{CrMo} 4-5$ constructional steel can be considered as an isotropic material, in this step of simulation average anisotropy energy density $K_{a n}$ was equal to 0 . On the base of differential evolution based simulation, the following parameters of the Jiles-Atherton model were achieved: $a=289 \mathrm{~A} / \mathrm{m}, k=296 \mathrm{~A} / \mathrm{m}$, $c=0.599, \alpha=4.15 \times 10^{-4}, M_{s}=1.661 \times 10^{5} \mathrm{~A} / \mathrm{m}$.
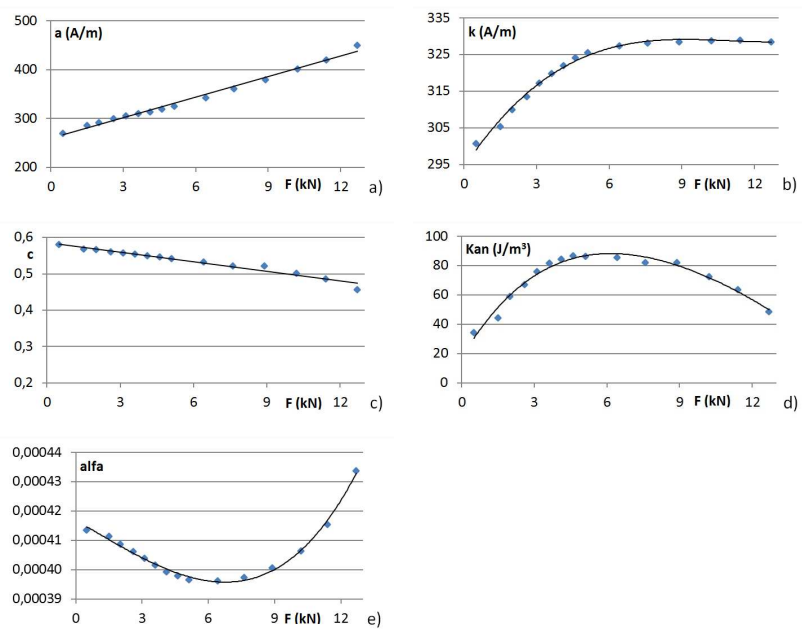

Fig. 3. Stress dependence of Jiles-Atherton model parameters: (a) a describing domain walls density, (b) $k$ describing the average energy required to break pinning, (c) $c$ magnetization reversibility, (d) $K_{a n}$ anisotropy energy density, (e) $\alpha$ describing inter domain coupling (trend line).

In the next step of simulation, the stress dependence of the Jiles-Atherton model parameters was determined on the base of gradient-less optimization process utilizing the Powell algorithm. For this step constant value of saturation magnetization $M_{s}$ was assumed due to the fact that saturation magnetization $M_{s}$ does not depend on mechanical stresses. Moreover, $\psi$ was assumed to be equal to 0 due to the fact that stresses are parallel to the direction of magnetization. The stress dependence of the Jiles-Atherton model parameters are presented in Fig. 3. Value of average anisotropy energy density $K_{a n}$ is connected with stress-induced anisotropy energy in magnetic material. 


\section{Results and conclusions}

Figures 4-6 present experimental results and the results of modelling for three selected values of magnetizing field. The figures clearly show the stress influence on the value of the flux density $B$. This dependence is well described by extended Jiles-Atherton model, which is confirmed by the fact that $R^{2}$ Pearson coefficient exceeds 0.93 for all values of amplitude of magnetizing field.

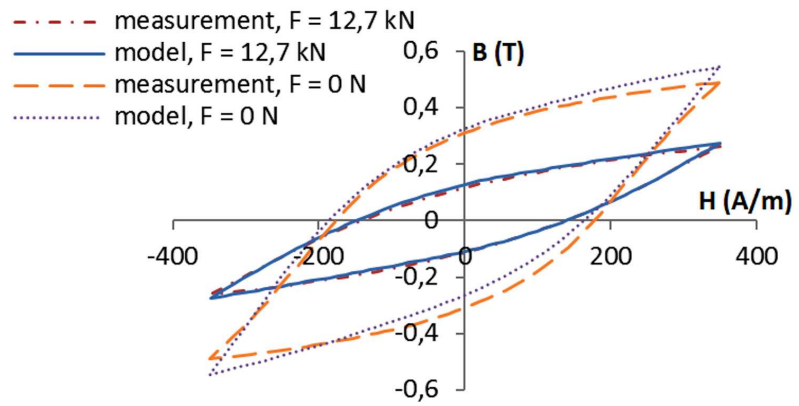

Fig. 4. Experimental results and the results of modelling, $H_{m}=350 \mathrm{~A} / \mathrm{m}$.

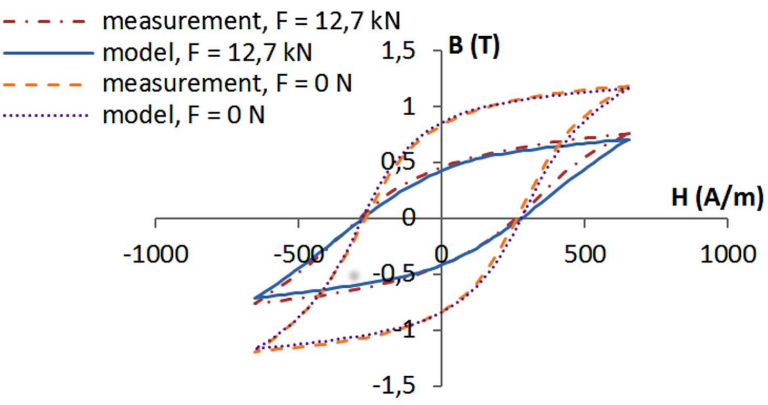

Fig. 5. Experimental results and the results of modelling, $H_{m}=655 \mathrm{~A} / \mathrm{m}$.

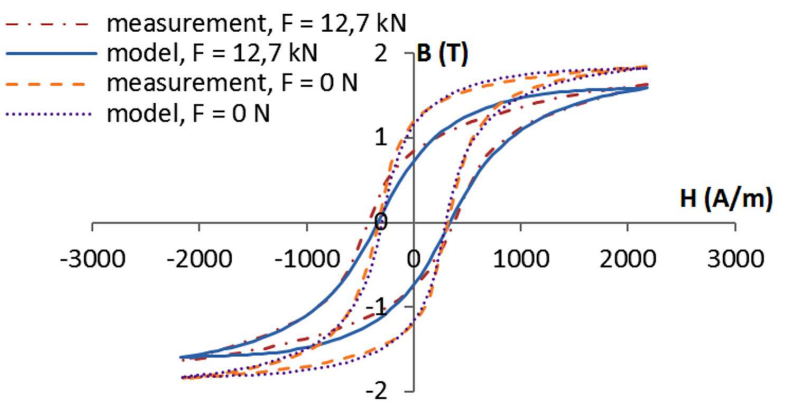

Fig. 6. Experimental results and the results of modelling, $H_{m}=2170 \mathrm{~A} / \mathrm{m}$.
Figure 3 presents the dependence of selected JilesAtherton model parameters. The values of the parameters $a$ describing domain wall density and parameter $k$ describing the average energy required to break pinning site increase monotonically. The value of the magnetization reversibility $c$ decreases monotonically. The value of the inter domain coupling $\alpha$ and anisotropy energy density $K_{a n}$ are following a parabolic curve. Saturation magnetisation $M_{s}$ is constant.

Results presented in this paper are filling the gap connected with modelling of stress influence of magnetic characteristic of steel trusses. As a conclusion presented results create the possibility practical use of quantitative magnetic hysteresis loop modelling to detect stress of construction elements.

\section{Acknowledgments}

This work was partially supported by the statutory funds of Institute of Metrology and Biomedical Engineering, Warsaw University of Technology (Poland).

\section{References}

[1] A. Bienkowski, R. Szewczyk, T. Kulik, J. Ferenc, J. Salach, J. Magn. Magn. Mater. 304, e624 (2006).

[2] W. Fuller Brown Jr., J. Appl. Phys. 36, 994 (1965).

[3] J. Salach, R. Szewczyk, P. Svec Sr., A. Bienkowski, D. Jackiewicz, P. Svec Jr., M. Nowicki, W. Winiarski, M. Kachniarz, M. Gruszecka, Acta Phys. Pol. A $\mathbf{1 2 7}$, 617 (2015).

[4] D.C. Jiles, D. Atherton, J. Magn. Magn. Mater. 61, 48 (1986).

[5] A. Ramesh, D.C. Jiles, Y. Bi, J. Appl. Phys. 81, 5585 (1997).

[6] R. Szewczyk, Materials 7, 5109 (2014).

[7] K. Chwastek, J. Szczygłowski, Math. Comput. Simulat. 71, 206 (2006).

[8] R. Biedrzycki, D. Jackiewicz, R. Szewczyk, J. Automat. Mobile Robot. Intellig. Syst. 8, 63 (2014). 